\title{
HLA DR alloantigens in different subsets of patients with Sjögren's syndrome and in family members
}

\author{
DEAN L. MANN ${ }^{1}$ AND HARALAMPOS M. MOUTSOPOULOS ${ }^{2 *}$ \\ From the 'Division of Cancer Biology and Diagnosis, National Cancer Institute, and ${ }^{2}$ Section of Clinical \\ Immunology, Laboratory of Microbiology and Immunology NIDR, NIH Bethesda, MD 20205, USA
}

SUMmaRY Patients with Sjögren's syndrome alone (Ss), Sjögren's syndrome with rheumatoid arthritis (Ss-RA), and Sjögren's syndrome with Raynaud's phenomenon (Ss-RP) were typed for the HLA DR and MT antigens. Ss-RA patients had higher frequencies of HLA DR4 than did Ss patients. HLA DR4 was also increased in frequency in patients with Ss-RP. This group of patients also showed increases in frequencies of HLA DR3. MT2 frequencies were elevated in all 3 patient groups, while MT1 was only increased in Ss. Three families with multiple individuals with Sjögren's syndrome were typed for HLA antigens. The affected individuals inherited unique combinations of haplotypes, suggesting the possibility of haplotype interaction in predisposition to disease.

Sjögren's syndrome is an autoimmune disease characterised by lymphoplasmocytic infiltration of exocrine glands and numerous organ and nonorgan-specific autoantibodies. ${ }^{1}$ The syndrome occurs alone (primary) or in association with other autoimmune rheumatic diseases (secondary), most commonly rheumatoid arthritis. ${ }^{2}$ Studies on HLA antigen associations in patients with Sjögren's syndrome have shown clear genetic similarities as well as differences in these 2 groups of patients. ${ }^{13}$ Clinical differences have also been described in patients with primary and secondary Sjögren's syndrome. More specifically, patients with primary Sjögren's syndrome more often have extraglandular manifestations than patients with secondary Sjögren's syndrome. ${ }^{4}$ Raynaud's phenomenon, an intermittent vasospasm of the digital arteries, occurs in approximately one-quarter of the patients with the primary disorder. ${ }^{5}$

This study shows that patients with primary Sjögren's syndrome and Raynaud's phenomenon have different HLA antigen associations compared with patients with primary Sjögren's syndrome without Raynaud's phenomenon and with patients with Sjögren's syndrome and rheumatoid arthritis. In addition from a study of 3 families with more than one member affected by Sjögren's syndrome we have

Accepted for publication 4 October 1982.

Correspondence to Dr Dean L. Mann, Building 37, Room 2C-11, NCI, NIH Bethesda, Maryland 20205, USA.

*Current address: Department of Medicine, School of Medicine, Ioannina, Greece. further studied the HLA associations with this disease. Although the previously described HLA associations are present, a more important association with combinations of HLA alloantigens is suggested.

\section{Materials and methods}

The study included 52 patients with Sjögren's syndrome. Forty patients had Primary Sjögren's syndrome (Ss) and 12 patients Sjögren's syndrome and rheumatoid arthritis (Ss-RA) (secondary Sjögren's syndrome). Fifteen out of the 40 patients with primary Sjögren's syndrome had Raynaud's phenomenon (Ss-RP).

Three families with a total of 19 individuals were studied. Seven of these individuals have primary Sjögren's syndrome. One individual has Sjögren's syndrome with rheumatoid arthritis (secondary Sjögren's syndrome).

The diagnosis of Sjögren's syndrome was based on xerostomia (decreased parotid flow rate and abnormal parotid scintigraphy) and keratoconjunctivitis sicca (punctate corneal ulcers on slitlamp examination and abnormal Schirmer's test). ${ }^{4}$ In all patients the diagnosis was confirmed by lip biopsy. ${ }^{6}$ All patients were Caucasian women between the ages of 20 and 50 years.

Typing for HLA A, HLA B, HLA C, HLA DR(w), $M T$, and $M B$ antigens has been described elsewhere. ${ }^{78}$ The antisera detecting the HLA DR(w), MT, and MB antigens were obtained from multiparous women and absorbed with pooled platelets to remove antibodies against HLA $\mathrm{A}, \mathrm{B}$, and $\mathrm{C}$ antigens. 
Antigen assignment was based on the correlation of reactivity of antisera in our testing panel with antigenic specificities assigned by the 8 th International Histocompatibility Testing Workshop and described in the proceedings.

\section{Results}

Table 1 compares the frequencies of HLA DR, MT1, and MT2 antigens in the 3 patient groups. HLA DR3 was elevated in frequency in patients with Ss and Ss-RP, and its frequency in the latter group differed significantly from that in patients with Ss-RA. The patients with Ss-RA and Ss-RP shared a common feature in that HLA DR4 was more frequent in these 2 than in Ss patients. MT1 was found in greatest frequency in the Ss patients, with a statistically significant difference from the frequency of this antigen in the Ss-RA patients. The frequency of MT1 in the Ss-RP group was nearly the same as that found in our normal population ( $48 \%$ ). MT2 was found in a relatively high frequency in all 3 patient groups compared with the frequency of $53 \%$ in our normal population. DR3 and 4 occurred together in 9 of 15 of the patients in the Ss-RP group and in no instance in patients in the other 2 groups.

Fig. 1 shows the results of HLA typing in family 1 . In this family the mother and one offspring were affected with the disease. The 2 individuals with Sjögren's syndrome were determined to share a common haplotype, $\mathrm{C}$, having the antigens HLA A3, B7, DRw6, and MT2. The maternal D haplotype was HLA A1, B17, DR7, and M1. The affected offspring had the A haplotype which was HLA A2, B44, DR2,

Table 1 Comparison of frequencies of HLA DR and MT antigens in patients with primary Sjögren's syndrome (Ss), Sjögren's syndrome with Raynaud's phenomenon (Ss-RP), and Sjögren's syndrome with rheumatoid arthritis (Ss-RA)

\begin{tabular}{lccc}
\hline & $\begin{array}{l}\text { Ss } \\
(25)^{*}\end{array}$ & $\begin{array}{c}\text { Ss-RP } \\
(15)^{*}\end{array}$ & $\begin{array}{c}\text { Ss-RA } \\
(12)^{*}\end{array}$ \\
\hline HLA & & $\%$ & \\
DR1 & 24 & 0 & 16 \\
DR2 & 7 & 6 & 8 \\
DR3 & 52 & $80 \dagger$ & 8 \\
DR4 & 12 & $73 \ddagger$ & $66 \ddagger$ \\
DR5 & 32 & 0 & 3 \\
DRw6 & 16 & 6 & 16 \\
DR7 & 16 & 2 & 16 \\
DRw8 & 4 & 0 & 8 \\
MT1 & 688 & 46 & 8 \\
MT2 & 80 & 93 & 92 \\
DR3 and 4 & 0 & 60 & 0 \\
\hline
\end{tabular}

"number of patients in each group.

tSignificant increase compared to Ss-RA, p<0.001.

fSignificant increase compared to Ss, $p<0.008$.

8Significant increase compared to Ss-RA, $p<0.003$.
Sjögren's Syndrome Family 1

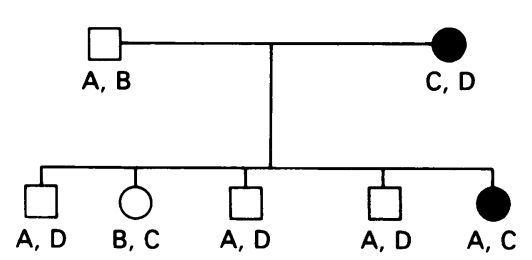
A. HLA A2 B44 Cw4 DR2 MT1
B. HLA A2 B40 CW1 DR2
C. HLA A3 B7 Cw- DRw6 MT2
D. HLAA1 B17 $\mathrm{CW}-\mathrm{DR7}$ MT1
Primary S.S.

Fig. 1 HLA types of family with 2 affected individuals with the diagnosis of primary Sjögren's syndrome.

and MT1. None of the siblings shared both HLA haplotypes of the affected individual. Both the affected individuals carried the MT1 antigens on one haplotype and the MT2 antigens on the other haplotype. The DR3 antigen was not found in this family.

The HLA haplotypes of family 2 are shown in Fig. 2 . In this family 3 individuals carried the diagnosis of Ss and one the diagnosis of Ss-RA. Two members of the family were unaffected. The parents in this family were deceased. All of the individuals affected with the primary disease had the $A$ and $D$ haplotypes. These haplotypes were determined to be; (A) HLA A1, B8, CW3, DR3, and MT2; and (D) HLA A11, Bw51, DR1, and MT1. The individual with secondary Sjögren's syndrome had the $A$ and $C$ haplotypes, the C haplotype having the HLA A2, B40, CW6, and DR4 antigens. The unaffected members had the BC and BD haplotypes. Note that the Ss affected members carried the MT1 and MT2 antigens on different haplotypes. In addition the A haplotype carried the DR3 antigen. The patient with Ss-RA

Sjögren's Syndrome Family 2

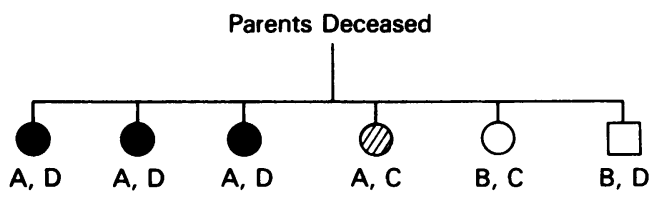

$\begin{array}{llcccl}\text { A. HLA A1 } & \text { B8 } & \text { Cw3 } & \text { DR3 } & \text { MT2 } & \text { Primary S.S. } \\ \text { B. HLA H3 } & \text { B18 } & - & \text { DR2 } & \text { MT1 } & \text { D Secondary S.S. } \\ \text { C. HLA 2 } & \text { B40 } & \text { Cw6 } & \text { DR4 } & - & \text { D } \\ \text { D. HLA A11 } & \text { B19 } & - & \text { DR1 } & \text { MT1 } & \end{array}$

Fig. 2 HLA types of family with 3 individuals with primary Sjögren's syndrome and one member with secondary disease. 
Sjögren's Syndrome Family 3

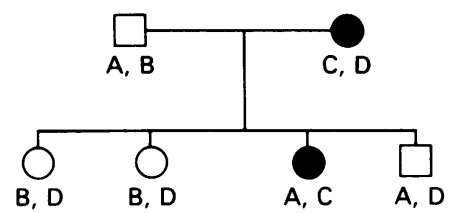

$\begin{array}{llcll}\text { A. HLA A1 } & \text { B8 } & \text { Cw3 } & \text { DR3 } & \text { MT2 } \\ \text { B. HLA A22 } & \text { B8 } & \text { Cw1 } & \text { DR3 } & \text { MT2 } \\ \text { C. HLA A23 } & \text { Bw38 } & - & \text { DRw6 } & \text { MT1 } \\ \text { D. HLA Aw34 } & \text { B27 } & - & \text { DR1 } & \text { MT2 }\end{array}$

Fig. 3 HLA types of family with 2 individuals with the diagnosis of primary Sjögren's syndrome.

inherited the $\mathrm{C}$ haplotype, which was carried by the DR4 antigen. This individual did not type for the MT1 antigen. The unaffected members of this family did not share the combinations of MT1, MT2, and DR3, DR4 that were found in the affected family members.

Fig. 3 shows the results of HLA typing in the third family with Ss. In this family the 2 affected individuals shared the C haplotype, HLA Aw23, Bw38, DRw6, and MT1. The maternal D haplotype carried the HLA antigens HLA Aw34, B27, DR1, and MT2. The affected daughter inherited the paternal haplotype A1, B8, CW3, DR3, and MT2. The affected members in this family, as in the previous families, typed for the MT1 determinant on one haplotype and the MT2 determinant on the other haplotype. None of the disease-free individuals in this family had this combination of antigens.

\section{Discussion}

The HLA DR3 antigen association with Ss is well documented. In addition we previously reported an increase in the DR4 antigen frequency (and decrease in DR3) in patients with Ss-RA. ${ }^{3}$ We now report an increased frequency of both HLA DR3 and HLA DR4 in patients with Ss-RP.

The recently described MT2 antigen was found with a relatively high frequency in all 3 patient groups. This increase is significantly different from an antigen frequency of $53 \%$, which was found in 205 normal Caucasians in our laboratory ( $\mathrm{Ss}, \mathrm{p}<0.02$; Ss-RP, $\mathrm{p}<0.008$; Ss- RA, $\mathrm{p}<0.02$ ). Comparison of the total frequency of MT2 in the 3 groups with the normal antigen frequency also demonstrated significant differences $(\mathrm{p}<0 \cdot 001)$. It would appear, therefore, that MT2 is the most frequent antigen controlled by the human major histocompatibility complex that is associated with Sjögren's syndrome regardless of other disease manifestations.
MT1 appears to be associated with Ss and infrequent in the Ss-RA group, the difference in frequency being statistically significant.

The combination of DR3 and DR4 was unique to the Ss-RP group. The results show that this group of patients shares antigenic determinants, one of which is common to each of the other groups.

The common increase of the antigen frequency of MT2 together with different DR determinants in these 3 patient groups that have a common disease manifestation suggests the possibility that gene interaction may play an important role in disease manifestation. This concept has been suggested by Svejgaard et al. ${ }^{9}$ in insulin-dependent diabetes and by Marcusson and Moller in psoriasis vulgaris. ${ }^{10}$ The results of our family studies enhance the credibility of this concept.

In the families studied it appears that combinations of antigens defining haplotypes are uniquely present in those individuals who have the disease. All the individuals with primary Sjögren's syndrome carried the MT1 antigen on one haplotype and the MT2 antigen on the other haplotype. Disease-free family members typed for one or another of these antigens but not the combination. In murine models combinations of $\mathrm{H} 2$ haplotypes appear to affect immune response $^{11}$ that is, certain immune response genes carried by one haplotype may have a profound effect on the regulation of immune response that is associated with another $\mathrm{H} 2$ haplotype. We suggest that combinations of haplotypes of the human major histocompatibility complex may be important in the disease processes that are manifest in Sjögren's syndrome as a primary disease or as a disease entity with other clinical manifestations.

\section{References}

1 Moutsopoulos H M, Chused T M, Mann D L, et al. Sjögren's syndrome (sicca syndrome): current issues. Ann Intern Med 1980; 92: 212-26.

2 Bloch K J, Buchanan W W, Wohl M J, Bunim J J. Sjögren's syndrome: clinical, pathological, and serological study of sixtytwo cases. Medicine (Baltimore) 1965; 44: 187-231.

3 Moutsopoulos H M, Mann D L, Johnson A H, Chused T M. Genetic differences between primary and secondary sicca syndrome. N Engl J Med 1979; 301: 761-3.

4 Moutsopoulos H M, Webber B L, Vlagopoulos T P, Chused T M, Decker J L. Difference in the clinical manifestations of sicca syndrome in the presence and absence of rheumatoid arthritis. Am J Med 1979; 66: 733-6.

5 Pavlidis N A, Karsh J Moutsopoulos H M. The clinical picture of primary Sjögren's syndrome: a retrospective study.J Rheumatol in press.

6 Tarpley T M, Anderson L G, White C L. Minor salivary gland involvement in Sjögren's syndrome. Oral Surg 1974; 37: 64-74.

7 Amos D B, Pool P. HLA typing. In: Rose N R, Friedman H, eds. Manual of clinical immunology. American Society of Microbiology, 1976.

8 Mann D L, Abelson L, Henkart P, Harris S. Amos D B 
Serologic detection of B lymphocyte antigens. In: Kissmeyer N, ed. Histocompatibility testing 1975. Copenhagen, Munksgaard, 1975: 707-9.

9 Svejgaard A, Platz P, Ryder L P, Staub-Nielsen L, Thompsen M. HLA-A and disease association: a survey. Transplant Rev 1975; 22: 3-43.
10 Marcusson J, Moller E. HLA antigens in a psoriatic family: comparative studies with MLC, HTC, PLT and serological HLA-DR determinants. Tissue Antigens 1976; 14: 279-95.

11 Berzofsky J A. Immune response genes in the regulation of mammalian immunity. In: Goldberger R F, ed. Biological regulation and development. New York: Plenum, 1980: 467-594. 